\title{
The potential role of advanced glycation end products (AGEs) and soluble receptors for AGEs (sRAGE) in the pathogenesis of adult-onset still's disease
}

Der-Yuan Chen ${ }^{1,2,3,4,7^{*}}$, Yi-Ming Chen ${ }^{1,5 \dagger}$, Chi-Chen Lin ${ }^{3 \dagger}$, Chia-Wei Hsieh ${ }^{3,5 \dagger}$, Yen-Ching Wu ${ }^{5}$, Wei-Ting Hung ${ }^{1,5}$, Hsin-Hua Chen ${ }^{1,5}$ and Joung-Liang Lan ${ }^{6}$

\begin{abstract}
Background: Accumulating evidence has demonstrated a pathogenic role of advanced glycation end products (AGEs) and receptors for AGEs (RAGE) in inflammation. Soluble RAGE (sRAGE), with the same ligands-binding capacity as full-length RAGE, acts as a "decoy" receptor. However, there has been scanty data regarding AGEs and sRAGE in adult-onset Still's disease (AOSD). This study aimed to investigate AGEs and sRAGE levels in AOSD patients and examine their association with clinical characteristics.

Methods: Using ELISA, plasma levels of AGEs and sRAGE were determined in 52 AOSD patients, 36 systemic lupus erythematosus(SLE) patients and 16 healthy controls(HC). Their associations with activity parameters and disease courses were evaluated.

Results: Significantly higher median levels of AGEs were observed in active AOSD patients $(16.75 \mathrm{pg} / \mathrm{ml})$ and active SLE patients $(14.80 \mathrm{pg} / \mathrm{ml})$ than those in HC $(9.80 \mathrm{pg} / \mathrm{ml}$, both $\mathrm{p}<0.001)$. AGEs levels were positively correlated with activity scores $(r=0.836, p<0.001)$, ferritin levels $(r=0.372, p<0.05)$ and CRP levels $(r=0.396, p<0.005)$ in AOSD patients. Conversely, significantly lower median levels of sRAGE were observed in active AOSD patients (632.2 pg/ $\mathrm{ml})$ and active SLE patients $(771.6 \mathrm{pg} / \mathrm{ml})$ compared with HC $(1051.7 \mathrm{pg} / \mathrm{ml}$, both $\mathrm{p}<0.001)$. Plasma sRAGE levels were negatively correlated with AOSD activity scores $(r=-0.320, p<0.05)$. In comparison to AOSD patients with monocyclic pattern, significantly higher AGEs levels were observed in those with polycyclic or chronic articular pattern. With treatment, AGEs levels declined while sRAGE levels increased in parallel with the decrease in disease activity.
\end{abstract}

Conclusion: The elevation of AGEs levels with concomitant decreased SRAGE levels in active AOSD patients, suggests their pathogenic role in AOSD.

Keywords: Advanced glycation end products (AGEs), Soluble receptor for AGEs (sRAGE), Pathogenesis, Adult-onset Still's disease (AOSD), Systemic lupus erythematosus (SLE)

\footnotetext{
* Correspondence: dychen@vghtc.gov.tw

${ }^{\dagger}$ Equal contributors

${ }^{1}$ Faculty of Medicine, National Yang-Ming University, Taipei, Taiwan

${ }^{2}$ Department of Medical Education and Research, Taichung Veterans General

Hospital, Taichung, Taiwan

Full list of author information is available at the end of the article
} 


\section{Background}

Advanced glycation end products (AGEs) result from non-enzymatic glycation and glycoxidation of proteins, lipids, or nucleic acids [1,2]. Inflammation leads to oxidative stress and the consequent formation of reactive carbonyl compounds, which are partly transformed into AGEs [3,4]. The increased accumulation of AGEs has been reported in pathophysiological situations such as Alzheimer's disease and inflammation [1-3]. The increased accumulation of AGEs has also been shown positively correlated with disease duration in systemic lupus erythematosus (SLE) [5-7]. These observations point to a pathogenic role of AGEs in inflammatory diseases.

Identified as a signal transduction receptor for AGEs $[8,9]$, the receptor for AGEs (RAGE) consists of an Nterminal V-type Ig-like domain, two tandem C-type domains, a single transmembrane domain, and a short C-terminal intracellular cytoplasmic tail [8]. Accumulating evidence shows that RAGE plays critical role in inflammatory processes $[10,11]$, and RAGE polymorphisms are associated with susceptibility and disease severity of lupus nephritis [12]. AGEs interact with both types of RAGE, namely full-length RAGE and Ctruncated RAGE [13-15]. The interaction of AGEs with full-length RAGE induces activation of intracellular signaling and results in secretion of cytokines such as TNF- $\alpha$, which plays a major role in inflammatory responses $[13,14]$.

RAGE also exists in soluble form, termed soluble Ctruncated RAGE (sRAGE), which lacks the transmembrane and cytosolic domains of the full-length RAGE. The sRAGE has the same AGEs-binding capacity as fulllength RAGE [16], and may act as a "decoy" receptor by binding ligands (such as AGEs) and preventing them from interacting with full-length RAGE $[17,18]$. Therefore, decreased sRAGE levels may enhance full-length RAGE signaling and thereby augment inflammation. A previous study demonstrated that rheumatoid arthritis (RA) patients displayed lower sRAGE levels compared to healthy subjects and osteoarthritis patients [19]. Recent studies also observed low sRAGE levels in patients with active inflammatory diseases, such as Takayasu's arteritis and SLE $[20,21]$.

Adult onset Still's disease (AOSD) is an inflammatory disorder, characterized by fever, rash, arthritis, multiorgans involvement, and increased acute phase reactants [22-26]. Although the aetiopathogenesis of AOSD remains unclear, cytokine-mediated inflammation may contribute to the development of this disease [27-29]. Therefore, we hypothesize that inflammatory responses in AOSD may be related to high AGEs levels and/or low sRAGE levels, which may lead to enhanced production of inflammatory mediators. However, there has been scanty data concerning circulating levels of AGEs and sRAGE in AOSD patients.

In this study, we examined plasma levels of AGEs and sRAGE in AOSD patients, SLE patients, and healthy subjects. Although AOSD has recently been considered as an autoinflammatory, instead of autoimmune, disease [30,31], we enrolled SLE patients as the disease control because both diseases share partial clinical manifestations, and the expression of AGEs or sRAGE has been observed in SLE [5,6,21]. The associations of AGEs and sRAGE levels with clinical characteristics, activity parameters, and disease courses were also investigated.

\section{Methods \\ Patients}

In this monocentric and prospective study, 52 patients with AOSD fulfilling the Yamaguchi criteria [32] were enrolled consecutively. Patients with infections, malignancies, or other rheumatic diseases were excluded. Disease activity of AOSD was assessed using a modified Pouchot score described by Rau et al. [33], and active AOSD was defined as a disease activity score of at least 3. All AOSD patients received corticosteroids and nonsteroidal anti-inflammatory drugs (NSAIDs). Besides, the disease-modifying anti-rheumatic drugs (DMARDs) prescribed included hydroxychloroquine (42 patients), methotrexate (40 patients), sulfasalazine (28 patients), and azathioprine (12 patients). Defined as described in previous studies [34,35], one of three patterns of disease course was determined for each AOSD patient. Thirty-six patients fulfilling the 1997 revised criteria of the American College of Rheumatology (ACR) for SLE [36] were included as disease controls. Disease activity of SLE was determined by calculating SLE disease activity index (SLEDAI) [37], and active SLE was defined as a SLEDAI score of at least 6. Sixteen healthy volunteers who had no rheumatic disease were the normal controls. History of cigarette smoking, including past and present smoking habit, was taken and body mass index (BMI) was calculated as body weight in kilograms divided by the square of body height in meters. To decrease the influence of foods on AGEs levels, we advised the participants to avoid foods high in AGEs, such as donuts and dark-colored soda for at least one week before starting the investigation. Venous blood samples taken in the early morning were centrifuged at $1000 \mathrm{~g}$ for $10 \mathrm{~min}$ within $15 \mathrm{~min}$ of withdrawal, and all the plasma samples had been stored at $-70^{\circ} \mathrm{C}$ until used simultaneously for the determination of levels of AGEs or sRAGE. To avoid the potential effects of hypoglycemic agents, statins, and angiotensin converting enzyme inhibitors (ACEI) on AGEs or sRAGE levels [38-40], subjects receiving these medications were excluded from this study. The ethics committee, the Institutional Review Board of Taichung Veterans General Hospital (CE11010), had approved the design of this study and 
the written consent was obtained according to the Declaration of Helsinki.

\section{Determination of plasma levels of AGEs and RAGE using ELISA}

Plasma levels of total AGEs, which include $\mathrm{N}^{\varepsilon}$-Carboxymethyllysine (CML), pentosidine, and other AGE structures, were determined in all subjects using ELISA (Cell Biolabs Inc., San Diego, CA, USA) according to the manufacturer's instructions. Plasma sRAGE levels were determined using another ELISA (R\&D Systems, Inc., Minneapolis, MN, USA) which detects total sRAGE pool. The minimum detectable sRAGE level is $4.12 \mathrm{pg} /$ $\mathrm{ml}$. All assays were performed with both inter- and intra-assay coefficient of variation $(\mathrm{CV})$ of less than $10 \%$.

\section{Statistical analysis}

The results are presented as the mean \pm SD or median (interquartile range). Kruskal-Wallis test was used for between-group comparison of AGEs or sRAGE levels. When this test showed significant differences, the exact p-value was then determined using the Mann-Whitney $\mathrm{U}$ test. The correlation coefficient was obtained through the nonparametric Spearman's rank correlation test. A logistic regression analysis was used to evaluate the effects of clinical characteristics and disease activity parameters on levels of AGEs or sRAGE in AOSD patients. Wilcoxon signed rank test was employed to compare the levels of AGEs and sRAGE during follow- up in AOSD patients after effective therapy. A probability of less than 0.05 was considered significant.

\section{Results}

Clinical characteristics of AOSD patients and SLE patients Among the 30 patients with active AOSD, spiking fever $\left(\geq 39^{\circ} \mathrm{C}\right)$, rash, arthritis, and lymphadenopathy were noted in 30 (100\%), 25 (83.3\%), 18 (60.0\%), and 7 (23.3\%) patients respectively. Twenty-five SLE patients had active disease (mean SLEDAI \pm SD, $12.00 \pm 1.11$ ), eleven (30.6\%) with renal involvement, and 11 had inactive disease (mean SLEDAI \pm SD, $4.11 \pm 0.63$ ) at the time of investigation. There were no significant differences in age at onset, proportion of females, BMI, or proportion of past or present smoker between AOSD patients and SLE patients or healthy subjects (Table 1).

Plasma levels of AGEs in AOSD patients and SLE patients As shown in Figure $1 \mathrm{~A}$ and Table 2, significantly higher AGEs levels were observed in active AOSD patients and active SLE patients than the levels in healthy subjects. Significantly higher AGEs levels were also observed in active AOSD patients compared with inactive AOSD patients or inactive SLE patients. However, there was no significant difference in AGEs levels between active AOSD patients and active SLE patients.

Table 1 Demographic data and clinical characteristics of patients with adult-onset Still's disease (AOSD), patients with systemic lupus erythematosus (SLE), and healthy controls (HC)\#

\begin{tabular}{|c|c|c|c|}
\hline & AOSD $(n=52)$ & SLE $(n=36)$ & $H C(n=16)$ \\
\hline Age at study entry (years) & $37.1 \pm 13.4$ & $38.0 \pm 12.1$ & $32.5 \pm 6.1$ \\
\hline Proportion of females & $37(71.2 \%)$ & $30(83.3 \%)$ & $10(62.5 \%)$ \\
\hline Serum creatinine (mg/dL) & $0.84 \pm 0.14$ & $0.96 \pm 0.92$ & $0.89 \pm 0.17$ \\
\hline Body mass index $\left(\mathrm{kg} / \mathrm{m}^{2}\right)$ & $22.6 \pm 2.7$ & $21.4 \pm 1.8^{*}$ & $21.8 \pm 1.7$ \\
\hline AOSD activity scores & $4.3 \pm 2.0$ & NA & NA \\
\hline CRP levels (normal <0.3 mg/dL) & $2.66 \pm 2.44$ & NA & NA \\
\hline Ferritin levels (normal <280 ㅆg/L) & $1458 \pm 4717$ & NA & NA \\
\hline SLEDAI & NA & $10.0 \pm 1.0$ & NA \\
\hline C3 (normal 84-160 mg/dL) & NA & $86.9 \pm 5.9$ & NA \\
\hline C4 (normal 12-36 mg/dL) & NA & $19.6 \pm 2.1$ & NA \\
\hline Anti-dsDNA (normal <92.7U/mL) & NA & $251.4 \pm 36.4$ & NA \\
\hline Nephritis & $0(0.0 \%)$ & $11(30.6 \%)^{* *}$ & NA \\
\hline Smoker (past) & $10(19.2 \%)$ & $7(19.4 \%)$ & $3(18.8 \%)$ \\
\hline Smoker (present) & $6(11.5 \%)$ & $5(13.9 \%)$ & $2(12.5 \%)$ \\
\hline Hypertension & $4(7.7 \%)$ & $9(25.0 \%)^{* \S}$ & $0(0.0 \%)$ \\
\hline
\end{tabular}

Data are presented as mean \pm SD or number (percentage); NA: not applicable.

CRP: C-reactive protein; SLEDAI: SLE disease activity index; C3: complement 3; C4: complement 4; Anti-ds DNA: anti-double strand DNA antibody; Nephritis is defined by persistent proteinuria ( $>0.5 \mathrm{~g} / 24$ hours) or pathological examination of renal biopsy specimens showing lupus nephritis. ${ }^{*} \mathrm{P}<0.05$, ${ }^{* *} \mathrm{p}<0.001$, vs. AOSD. $\S p<0.001$, vs. Healthy controls. 

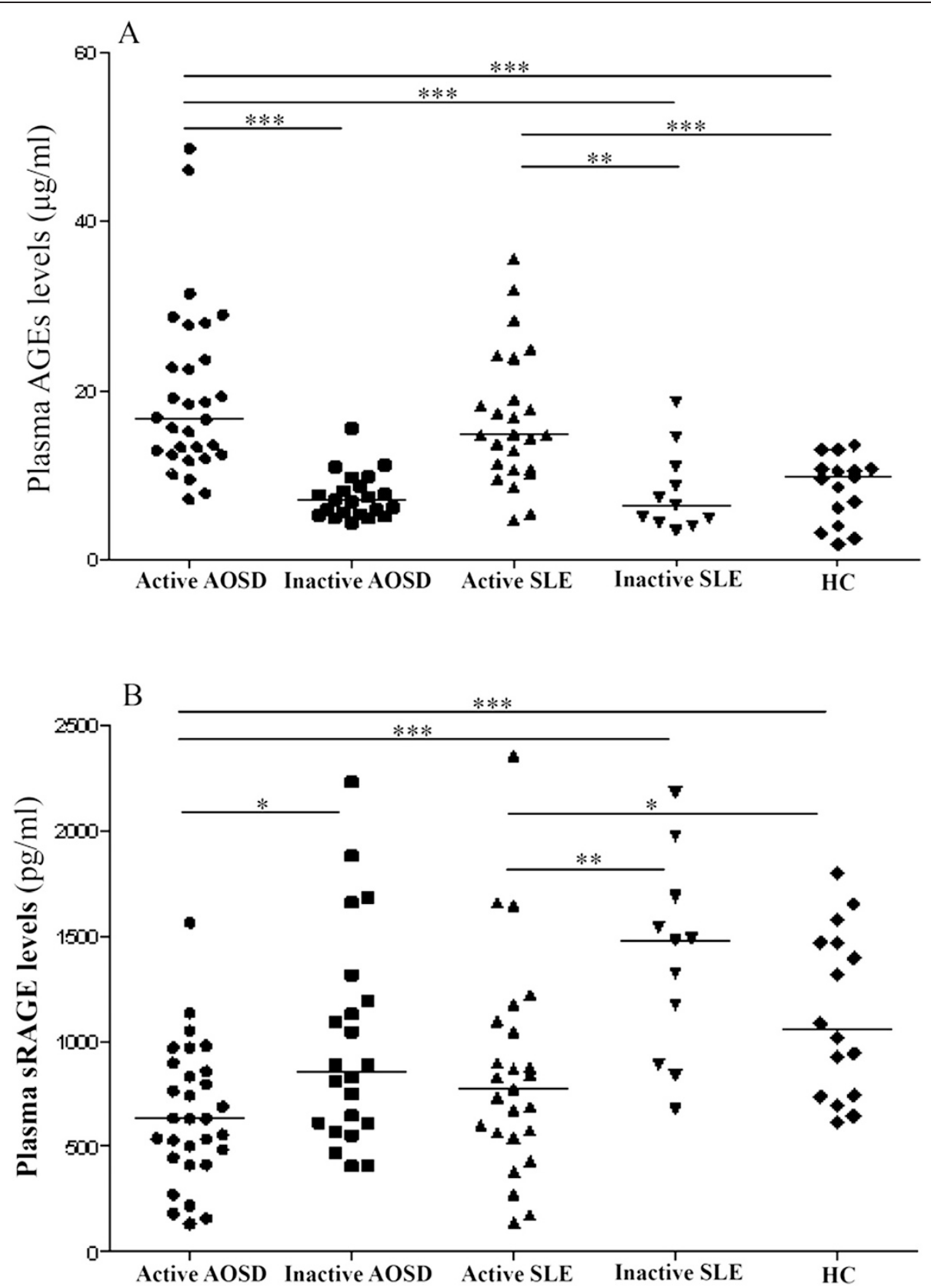

Figure 1 Comparison of plasma levels of (A) AGEs and (B) soluble RAGE (sRAGE) among 30 patients with active adult-onset Still's disease (AOSD), 22 patients with inactive AOSD, 25 patients with active systemic lupus erythematosus (SLE), 11 patients with inactive SLE, and 16 healthy subjects (HC). The horizontal line indicates median value for each group. ${ }^{*} p<0.05,{ }^{* *} p<0.005$, ${ }^{* *} p<0.001$, was determined by Mann-Whitney $U$ test.

Plasma levels of sRAGE in AOSD patients and SLE patients As shown in Figure $1 \mathrm{~B}$ and Table 2, significantly lower sRAGE levels were observed in active AOSD patients and active SLE patients compared with healthy subjects. Active AOSD patients also had significantly lower sRAGE levels than inactive AOSD patients. However, there was no significant difference in sRAGE levels between active AOSD patients and active SLE patients.
Correlation between disease activity parameters and AGEs levels or sRAGE levels

Plasma AGEs levels were positively correlated with disease activity parameters including clinical activity scores $(\mathrm{r}=0.836, \mathrm{p}<0.001)$, serum ferritin levels $(\mathrm{r}=0.372, \mathrm{p}<$ 0.05), and CRP levels $(\mathrm{r}=0.396, \mathrm{p}<0.005)$ in AOSD patients. Similarly, plasma AGEs levels were positively correlated with SLEDAI $(\mathrm{r}=0.831, \mathrm{p}<0.001)$ in SLE patients. In contrast, plasma sRAGEs levels were 
Table 2 Comparison of plasma AGEs levels and plasma SRAGE levels in 52 patients with adult-onset Still's disease (AOSD), 36 patients with systemic lupus erythematosus (SLE), and 16 healthy subjects (HC)

\begin{tabular}{ll}
\hline AGEs and sRAGE, patient groups & Median (interquartile range) \\
\hline AGEs levels, $\mu \mathrm{g} / \mathrm{mL}$ & \\
Active AOSD $(n=30)$ & $16.75(12.45-24.66)^{* * \# \# \S \S}$ \\
Inactive AOSD $(n=22)$ & $7.02(5.43-9.01)$ \\
Active SLE $(n=25)$ & $14.80(10.68-21.42)^{* * \# \# \S}$ \\
Inactive SLE $(n=11)$ & $6.49(4.40-11.10)$ \\
HC $(n=16)$ & $9.80(4.55-10.83)$ \\
SRAGE levels, pg/mL & \\
Active AOSD $(n=30)$ & $632.2(437.8-866.9)^{* * \# \S \S \S}$ \\
Inactive AOSD $(n=22)$ & $858.9(599.8-1222.2)^{\S}$ \\
Active SLE $(n=25)$ & $771.6(553.0-1069.1)^{* \S \S}$ \\
Inactive SLE $(n=11)$ & $1480.0(892.1-1686.9)$ \\
HC $(n=16)$ & $1051.7(738.2-1468.3)$
\end{tabular}

AGEs: advanced glycation end products; sRAGE: soluble receptor for AGEs. ${ }^{*} p<0.05,{ }^{* *} p<0.001$, versus $\mathrm{HC} ;{ }^{*} p<0.05,{ }^{\# \#} \mathrm{p}<0.001$ versus inactive AOSD; ${ }^{5} \mathrm{p}<0.05,{ }^{55} \mathrm{p}<0.005,{ }^{\S 55} \mathrm{p}<0.001$, versus inactive SLE.

Mann-Whitney $U$ test was used for between-group comparison of numerical variables.

inversely correlated with clinical activity scores $(r=-0.320$, $\mathrm{p}<0.05)$ in AOSD patients, as well as SLEDAI $(\mathrm{r}=-0.483$, $\mathrm{p}<0.005)$ in SLE patients. In addition, there was an inverse correlation between AGEs levels and sRAGE levels in AOSD patients $(\mathrm{r}=-0.323, \mathrm{p}<0.05)$ and SLE patients $(\mathrm{r}=-0.454, \mathrm{p}<0.01)$.

\section{Logistic regression analysis}

Using univariate regression analysis, AGEs levels were positively associated with the presence of anemia and disease activity parameters such as CRP levels and ferritin levels in AOSD patients (Table 3). Plasma sRAGE levels were negatively associated with the presence of anemia and leukocytosis in AOSD patients. Using univariate and multivariate analysis, AGEs levels were positively correlated with activity scores, while sRAGE levels were negatively correlated with activity scores in AOSD patients.

The differences in plasma levels of AGEs and sRAGE in AOSD patients with different patterns of disease course Among all AOSD patients, 28 (53.8\%) had polycyclic systemic pattern, 12 (23.1\%) had monocyclic systemic pattern, and the remaining $12(23.1 \%)$ had chronic articular pattern. As illustrated in Figure 2, significantly higher AGEs levels were observed in AOSD patients with polycyclic pattern or chronic articular pattern compared with those with monocyclic pattern. In contrast, significantly lower sRAGE levels were demonstrated in
Table 3 Logistic regression analysis of plasma AGEs levels and sRAGE levels in patients with adult-onset Still's disease (AOSD)

\begin{tabular}{lllllll}
\hline $\begin{array}{l}\text { AOSD patients } \\
(\mathbf{n}=\mathbf{5 2})\end{array}$ & \multicolumn{2}{l}{ AGEs levels } & \multicolumn{5}{l}{ sRAGE levels } \\
\cline { 2 - 7 } & $\mathbf{B}$ & Beta & $\mathbf{p}$ & $\mathbf{B}$ & Beta & $\mathbf{p}$ \\
\hline Univariate analysis & & & & & & \\
$\quad$ Smoking & -1.00 & -0.03 & 0.806 & 30.79 & 0.02 & 0.864 \\
BMI & -1.01 & -0.28 & 0.056 & 23.07 & 0.14 & 0.314 \\
Leukocytosis & 5.31 & 0.27 & 0.055 & -313.40 & -0.36 & 0.010 \\
Anemia & 6.89 & 0.30 & 0.032 & -308.35 & -0.30 & 0.031 \\
CRP & 2.21 & 0.55 & 0.000 & -8.63 & -0.05 & 0.734 \\
Log(Ferritin) & 10.18 & 0.58 & 0.000 & -0.02 & -0.16 & 0.244 \\
Activity score & 3.81 & 0.77 & 0.000 & -85.88 & -0.39 & 0.004 \\
Multivariate analysis & & & & & & \\
Activity score & 2.63 & 0.53 & 0.000 & -45.56 & -0.21 & 0.062 \\
\hline
\end{tabular}

AGEs: advanced glycation end products; sRAGE: soluble receptor for AGEs; BMI: body mass index; CRP: C-reactive protein.

Leukocytosis is defined as white cell count $>10,000$ /cumm; anemia is defined as hemoglobin $\leqq 11.3 \mathrm{gm} / \mathrm{dl}$.

AOSD patients with polycyclic pattern than those in patients with monocyclic pattern.

\section{Changes in plasma levels of AGEs and sRAGE in AOSD patients after therapy}

Sixteen AOSD patients were available for examination both in the active phase and in the inactive phase after 6 months of treatment. As shown in Figure $2 \mathrm{C}$ and D, AGEs levels significantly declined (mean \pm SEM, $24.6 \pm$ $2.7 \mathrm{pg} / \mathrm{mL}$ vs. $11.2 \pm 1.6 \mathrm{pg} / \mathrm{mL}, \mathrm{p}<0.001)$ while sRAGE levels increased $(460.5 \pm 55.8 \mathrm{pg} / \mathrm{mL}$ vs. $794.9 \pm 77.0 \mathrm{pg} /$ $\mathrm{mL}, \mathrm{p}<0.001)$ significantly, paralleling the decreases in clinical activity scores $(5.88 \pm 0.41$ vs. $2.00 \pm 0.20, \mathrm{p}<$ $0.001)$ and CRP levels $(4.07 \pm 0.63 \mathrm{pg} / \mathrm{ml}$ vs. $0.97 \pm$ $0.26 \mathrm{pg} / \mathrm{ml}, \mathrm{p}<0.001)$ in AOSD patients after 6-month therapy.

\section{Discussion}

This is the first study to examine plasma levels of AGEs and sRAGE in AOSD patients, and to characterize their association with activity parameters of this disease. Plasma AGEs levels were significantly higher in active AOSD patients compared with inactive AOSD patients or healthy subjects, with the levels positively correlated with activity parameters. In contrast, plasma sRAGE levels were significantly lower in active AOSD patients compared with inactive AOSD patients or healthy subjects, with their levels negatively correlated with activity parameters. Moreover, the decrease in AGEs levels and an increase in SRAGE levels paralleled disease remission. These observations indicate that overproduction of AGEs and a reverse regulation of sRAGE may be 

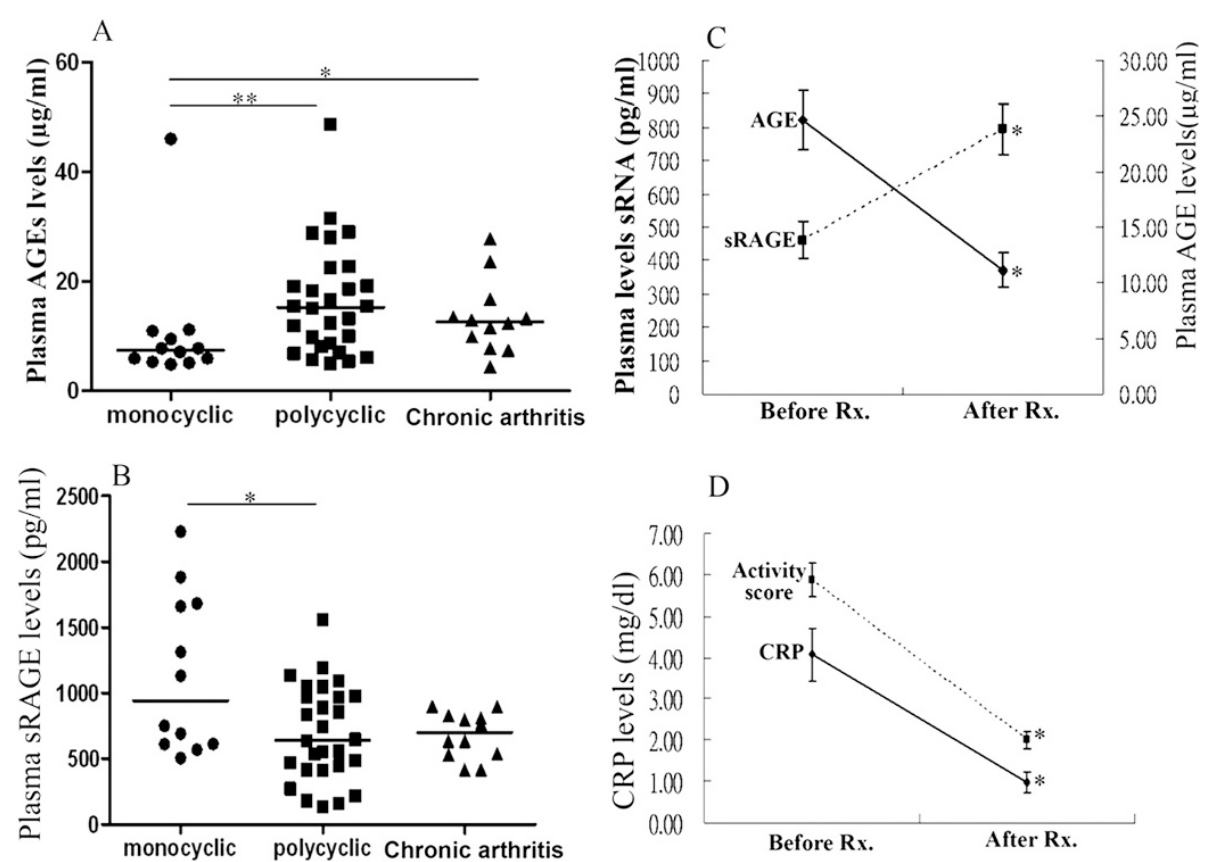

Figure 2 Comparison of plasma levels of (A) AGEs and (B) soluble RAGE (SRAGE) among AOSD patients with different patterns of disease courses, including 12 patients with monocyclic pattern, 28 patients with polycyclic pattern, and 12 patients with chronic arthritis. The horizontal line indicates median value for each group. ${ }^{*} p<0.05,{ }^{*} p<0.005$, was determined by Mann-Whitney $U$ test. The changes in (C) plasma levels of AGEs as well as SRAGE and (D) disease activity parameters including clinical activity scores and C-reactive protein (CRP) levels in 16 AOSD patients after 6 months of treatment. Rx:: treatment. Data are presented as mean \pm SEM. ${ }^{*} p<0.005$, versus before treatment, determined by the Wilcoxon signed rank test.

involved in AOSD pathogenesis, and may act as activity indicators of this disease.

RAGE, the most characterized receptor of AGEs, appears to play an important role in inflammatory responses [9-12]. Soluble RAGE (sRAGE), the extracellular domain of RAGE, is generated by two distinct mechanisms, the splice variant [endogenous secretory RAGE (esRAGE)] secretion and cleavage of membrane-bound RAGE (cRAGE) through metalloproteinase [15,16]. Both esRAGE and cRAGE are functionally equivalent and interact with the same RAGE ligands. The sRAGE acts as a decoy receptor, and effectively blocks the binding of AGEs or other ligands to cell membrane-bound RAGE $[17,18]$, which may explain the inverse correlation between sRAGE levels and AGEs levels in AOSD patients. The reduction of sRAGE levels may also represent an inadequate protective response in inflammatory diseases. Likewise, circulating sRAGE levels have been shown to be decreased in patients with RA [19], Takayasu's arteritis [20], SLE [21], and Still's disease [41]. In agreement with previous findings that decreased sRAGE levels were negatively correlated with disease activity in patients with Still's disease [41], we similarly demonstrated significantly lower sRAGE levels in active AOSD patients than those in quiescent AOSD patients, with an inverse correlation between plasma sRAGE levels and disease activity. Therefore, plasma sRAGE level may serve as a biomarker and negative regulator of inflammation [42], and the administration of recombinant sRAGE has been of therapeutic use in inflammatory murine models $[43,44]$.

As with other previous findings [5,6,21] and similar to AOSD patients, our SLE patients also had increased AGEs levels which were positively correlated with SLEDAI. On the other hand, active SLE patients had decreased plasma sRAGE levels negatively correlated with disease activity, substantiating the findings that treatment with sRAGE significantly improved nephritis and histologic renal damage in lupus-prone (NZB/NZW) mice [45].

There are two possible mechanisms that may explain the low sRAGE levels in our active AOSD patients. One is that plasma sRAGE levels may be modulated by its binding with inflammatory ligands, thereby promoting consumption of sRAGE [46]. Compatible with this hypothesis, an elevated AGEs levels may increase the binding and consumption of sRAGE in this study. The other is that the reduced production of endogenous secretory sRAGE may not be sufficient as a decoy receptor for binding inflammatory ligands and therefore there is weakened protection against the inflammatory response. The inadequate levels of sRAGE might amplify inflammatory responses by increasing the engagement of accumulated AGEs on cell-bound RAGE [19]. 
Interestingly, a logistic regression analysis demonstrated that plasma AGEs levels were positively associated with the presence of leukocytosis, whereas sRAGE levels showed negative association. Because cell-bound RAGE may function as a counter-receptor for leukocyte integrin Mac-1 and was directly involved in leukocyte recruitment, sRAGE could act as a potential inhibitor of leukocyte recruitment [47]. In addition, we found that the presence of anemia in AOSD patients was positively associated with AGEs levels, but negatively associated with sRAGE levels, which resonates with the findings that plasma AGEs, a marker of oxidative stress, was a predictor of anemia in older community-dwelling adults [48].

The disease courses of AOSD patients may vary considerably. Those with polycyclic disease pattern exhibit relapsing episodes with systemic inflammation superimposed on chronic low-grade inflammation. It is interesting to note that significantly higher AGEs levels were observed in AOSD patients with polycyclic pattern or chronic arthritis compared with those with monocyclic pattern. Cumulative inflammation may contribute to the increased AGEs levels in AOSD patients with polycyclic pattern or chronic arthritis, as with the enhanced AGEs levels positively related to the disease duration of SLE [5], and the elevation of AGEs levels in RA patients [3]. In contrast, plasma sRAGE levels were lower in AOSD patients with polycyclic pattern than in those with monocyclic pattern. Thus, we hypothesize that the dysregulated inflammation in patients with polycyclic pattern may persist via insufficient inhibition by sRAGE.

Longitudinal follow-up of our AOSD patients showed that AGEs levels declined while sRAGE levels increased with therapy, paralleling the reduction of disease activity. The change in sRAGE levels after 6-month therapy was similar to a recent finding that sRAGE levels increased after long-term (more than one month) treatment [21], and further supports the potential for AGEs or sRAGE as a biomarker of disease activity [42].

There were some limitations in our study. Because AGEs represent a heterogeneous group of compounds, future studies are needed to determine the major compound which is related to AOSD. The sRAGE may act as a decoy receptor; however, we still did not elucidate the functional role of sRAGE in AOSD. Our preliminary results await further substantiation by ex vivo studies which will determine interleukin-1 secretion by subjects' monocytes treated with AGEs or sRAGE.

\section{Conclusions}

In conclusion, increased AGEs as well as decreased sRAGE, which were significantly correlated with disease activity parameters, may be involved in AOSD pathogenesis. We provide the first evidence showing that plasma levels of AGEs and sRAGE may be linked with disease outcome in AOSD. The results could be of translational interest, and may potentially lead to the discovery of novel activity biomarkers [42] or promising therapeutic targets such as sRAGE, which has been used to protect against colonic inflammation or autoimmune encephalitis $[43,44]$.

\section{Key messages}

1. Elevated AGEs levels and decreased sRAGE levels were observed in both AOSD and SLE.

2. Associations of AGEs and sRAGE levels with disease activity indicate their involvement in pathogenesis.

\section{Abbreviations}

ACEl: Angiotensin converting enzyme inhibitors; ACR: American College of Rheumatology; AGEs: Advanced glycation end products; AOSD: Adult onset Still's disease; BMI: Body mass index; CRAGE: Cleavage of membrane-bound RAGE; CV: Coefficient of variation; DMARDs: Disease-modifying anti-rheumatic drugs; esRAGE: Endogenous secretory RAGE; NSAIDs: Non-steroidal anti-inflammatory drugs; RAGE: Receptor for AGEs; SLE: Systemic lupus erythematosus; SLEDAI: SLE disease activity index; sRAGE: Soluble C-truncated RAGE.

\section{Competing interests}

The authors declare that they have no competing interests.

\section{Authors' contributions}

All authors made substantive intellectual contributions to the present study and approved the final manuscript. D-YC conceived of the study, generated the original hypothesis, designed the study, acquired clinical data, conducted data analysis, and drafted and revised the manuscript. Y-MC, C-CL, and C-WH acquired clinical data, performed data analysis, and revised the manuscript. Y-CW conducted the experiment, performed data analysis, and drafted the manuscript. W-TH, H-HC, and J-LL performed clinical assessments of the study subjects, acquired clinical data, and revised the manuscript.

\section{Acknowledgements}

This work was supported by a grant from Taichung Veterans General Hospital (TCVGH-1010101C). The authors thank the Biostatistics Task Force of Taichung Veterans General Hospital, Taichung, Taiwan.

\section{Author details}

${ }^{1}$ Faculty of Medicine, National Yang-Ming University, Taipei, Taiwan. ${ }^{2}$ Department of Medical Education and Research, Taichung Veterans General Hospital, Taichung, Taiwan. ${ }^{3}$ Institute of Biomedical Science, National Chung-Hsing University, Taichung, Taiwan. ${ }^{4}$ Institute of Microbiology and Immunology, Chung-Shan Medical University, Taichung, Taiwan. ${ }^{5}$ Division of Allergy, Immunology and Rheumatology, Taichung Veterans General Hospital, Taichung, Taiwan. 'Division of Immunology and Rheumatology, China Medical University Hospital, Taichung, Taiwan. 7 Division of Allergy, Immunology and Rheumatology, Department of Internal Medicine, Taichung Veterans General Hospital, No. 160, Sec. 3, Chung-Kang Rd., Taichung 407, Taiwan.

Received: 23 September 2014 Accepted: 29 April 2015 Published online: 09 May 2015

\section{References}

1. Smith MA, Taneda S, Richey PL, Miyata S, Yan SD, Stern D, et al. Advanced Maillard reaction end products are associated with Alzheimer disease pathology. Proc Natl Acad Sci U S A. 1994;91:5710-4.

2. Anderson MM, Requena JR, Crowley JR, Thorpe SR, Heinecke JW. The myeloperoxidase system of human phagocytes generates nepsilon(carboxymethyl) lysine on proteins: a mechanism for producing advanced glycation end products at sites of inflammation. J Clin Invest. 1999;104:103-13. 
3. Vytasek R, Sedova L, Vilim V. Increased concentration of two different advanced glycation end-products detected by enzyme immuno- assays with new monoclonal antibodies in sera of patients with rheumatoid arthritis. BMC Musculoskelet Disord. 2010;11:83.

4. Li J, Hou F, Guo Z, Shan Y, Zang X, Liu Z. Advanced glycation end products upregulate $\mathrm{C}$-reactive protein synthesis by human hepatocytes through stimulation of monocyte IL-6 and IL-1 beta production. Scand J Immunol. 2007;66:555-62.

5. de Leeuw K, Graaff R, de Vries R, Dullaart RP, Smit AJ, Kallenberg CG, et al. Accumulation of advanced glycation endproducts in patients with systemic lupus erythematosus. Rheumatology (Oxford). 2007:46:1551-6.

6. Nienhuis $H L$, de Leeuw K, Bijzet J, Smit A, Schalkwijk CG, Graaff R, et al. Skin autofluorescence is increased in systemic lupus erythematosus but is not reflected by elevated plasma levels of advanced glycation endproducts. Rheumatology (Oxford). 2008;47:1554-8.

7. Kurien BT, Scofield RH. Autoimmunity and oxidatively modified autoantigens. Autoimmun Rev. 2008;7:567-73.

8. Neeper M, Schmidt AM, Brett J, Yan SD, Wang F, Pan YC, et al. Cloning and expression of RAGE: a cell surface receptor for advanced glycosylation end products of proteins. J Biol Chem. 1992;267:14998-5004.

9. Schmidt AM, Yan SD, Yan SF, Stern DM. The biology of the receptor for advanced glycation end products and its ligands. Biochim Biophys Acta. 2000;1498:99-111.

10. Mosquera JA. Role of the receptor for advanced glycation end products (RAGE) in inflammation. Invest Clin. 2010;51:257-68.

11. Lin L, Park S, Lakatta EG. RAGE signaling in inflammation and arterial aging Front Biosci. 2009;14:1403-13.

12. Martens HA, Nienhuis HL, Gross S, van der Steege G, Brouwer E, Berden JH, et al. Receptor for advanced glycation end products (RAGE) polymorphisms are associated with systemic lupus erythematosus and disease severity in lupus nephritis. Lupus. 2012;21:959-68.

13. Schmidt AM, Yan SD, Yan SF, Stern DM. The multiligand receptor RAGE as a progression factor amplifying immune and inflammatory responses. J Clin Invest. 2001;108:949-55.

14. Basta G, Lazzerini G, Massaro M, Simoncini T, Tanganelli P, Fu C, et al. Advanced glycation end products activate endothelium through signal-transduction receptor RAGE: a mechanism for amplification of inflammatory responses. Circulation. 2002;105:816-22.

15. Yonekura H, Yamamoto Y, Sakurai S, Petrova RG, Abedin MJ, Li H, et al. Novel splice variants of the receptor for advanced glycation end-products expressed in human vascular endothelial cells and pericytes, and their putative roles in diabetes-induced vascular injury. Biochem J. 2003:370:1097-109.

16. Raucci A, Cugusi S, Antonelli A, Barabino SM, Monti L, Bierhaus A, et al. A soluble form of the receptor for advanced glycation endproducts (RAGE) is produced by proteolytic cleavage of the membrane-bound form by the sheddase a disintegrin and metalloprotease 10 (ADAM10). FASEB J. 2008;22:3716-27.

17. Hanford LE, Enghild JJ, Valnickova Z, Petersen SV, Schaefer LM, Schaefer TM, et al. Purification and characterization of mouse soluble receptor for advanced glycation end products (sRAGE). J Biol Chem. 2004:279:50019-24.

18. Geroldi D, Falcone C, Emanuele E. Soluble receptor for advanced glycation end products: from disease marker to potential therapeutic target. Curr Med Chem. 2006;13:1971-8

19. Pullerits R, Bokarewa M, Dahlberg L, Tarkowski A. Decreased levels of soluble receptor for advanced glycation end products in patients with rheumatoid arthritis indicating deficient inflammatory control. Arthritis Res Ther. 2005; 7:R817-24

20. Mahajan N, Dhawan V, Malik S, Jain S. Serum levels of soluble receptor for advanced glycation end products (sRAGE) in Takayasu's arteritis. Int J Cardiol. 2010;145:589-91.

21. $\mathrm{Ma} C Y, M a J L$, Jiao $Y L$, Li JF, Wang LC, Yang QR, et al. The plasma level of soluble receptor for advanced glycation end products is decreased in patients with systemic lupus erythematosus. Scand J Immunol. 2012;75:614-22.

22. Ohta A, Yamaguchi M, Kaneoka H, Nagayoshi T, Hiida M. Adult Still's disease: review of 228 cases from the literature. J Rheumatol. 1987;14:1139-46.

23. Gerfaud-Valentin $M$, Jamilloux $Y$, Iwaz J, Sève P. Adult-onset Still's disease. Autoimmun Rev. 2014;13:708-22.

24. Kadavath S, Efthimiou P. Adult-onset Still's disease-pathogeensis, clinical manifestations, and new treatment options. Ann Med. 2015;22:1-9.
25. Narula N, Narula T, Abril A. Swizing the clinical presentation in adult onset Still's disease. An extensive literature review. Autoimmun Rev. 2015. [Epub ahead of print].

26. Ruscitti $P$, Cipriani $P$, Di Benedetto P, Ciccia F, Liakouli V, Carubbi F, et al. Increased level of $\mathrm{H}$-ferritin and its imbalance with $\mathrm{L}$-ferritin in bone marrow and liver of patients with adult onset Still's disease, developing macrophage activation syndrome, correlate with the severity of the diseases. Autoimmun Rev. 2015. [Epub ahead of print].

27. Kawashima M, Yamamura M, Taniai M, Yamauchi H, Tanimoto T, Kurimoto $M$, et al. Levels of interleukin-18 and its binding inhibitors in the blood circulation of patients with adult-onset Still's disease. Arthritis Rheum. 2001;44:550-60.

28. Choi JH, Suh CH, Lee YM, Suh YJ, Lee SK, Kim SS, et al. Serum cytokine profiles in patients with adult onset Still's disease. J Rheumatol. 2003;30:2422-7.

29. Chen DY, Lan JL, Lin FJ, Hsieh TY. Proinflammatory cytokine profiles in the sera and in the pathological tissues of patients with active untreated adult onset Still's disease. J Rheumatol. 2004;31:2189-98.

30. Kastner DL, Aksentijevich I, Goldbach-Mansky R. Autoinflammatory disease reloaded: a clinical perspective. Cell. 2010;140:784-90.

31. Rossi-Semerano L, Koné-Paut I. Is still's disease an autoinflammatory syndrome? Int J Inflam. 2012;2012:480373.

32. Yamaguchi M, Ohta A, Tsunematsu T, Kasukawa R, Mizushima Y, Kashiwagi $\mathrm{H}$, et al. Preliminary criteria for classification of adult Still's disease. J Rheumatol. 1992;19:424-30.

33. Rau M, Schiller M, Krienke S, Heyder P, Lorenz H, Blank N. Clinical manifestations but not cytokine profiles differentiate adult-onset Still's disease and sepsis. J Rheumatol. 2010;37:2369-76.

34. Cush JJ, Medsger TA, Christy WC, Herbert DC, Cooperstein LA. Adult-onset Still's disease: clinical course and outcome. Arthritis Rheum. 1987;30:186-94

35. Gerfaud-Valentin M, Maucort-Boulch D, Hot A, Iwaz J, Ninet J, Durieu I, et al. Adult-onset Still's disease: manifestations, treatment, outcome, and prognostic factors in 57 patients. Medicine (Baltimore). 2014;93:91-9.

36. Hochberg MC. Updating the American college of rheumatology revised criteria for the classification of systemic lupus erythematosus. Arthritis Rheum. 1997;40:1725.

37. Bombaridier C, Gladman DD, Urowitz MB, Caron D, Chang CH. The committee on prognosis studies in SLE. Derivation of the SLEDAl: a disease activity index for lupus patients. Arthritis Rheum. 1992;35:630-40

38. Nangaku M, Miyata T, Sada T, Mizuno M, Inagi R, Ueda Y, et al. Anti-hypertensive agents inhibit in vivo the formation of advanced glycation end products and improve renal damage in a type 2 diabetic nephropathy rat model. J Am Soc Nephrol. 2003;14:1212-22.

39. Quade-Lyssy P, Kanarek AM, Baiersdörfer M, Postina R, Kojro E. Statins stimulate the production of a soluble form of the receptor for advanced glycation end products. J Lipid Res. 2013;54:3052-61.

40. Tanji N, Markowitz GS, Fu C, Kislinger T, Taguchi A, Pischetsrieder M, et al. Expression of advanced glycation end products and their cellular receptor RAGE in diabetic nephropathy and nondiabetic renal disease. J Am Soc Nephrol. 2000;11:1656-66.

41. Myles A, Viswanath $V$, Singh YP, Aggarwal A. Soluble receptor for advanced glycation end products is decreased in patients with juvenile idopathic arthritis (ERA category) and inversely corelated with disease activity and S100A12 levels. J Rheumatol. 2011;38:1994-9.

42. Maillard-Lefebvre H, Boulanger E, Daroux M, Gaxatte C, Hudson BI, Lambert M. Soluble receptor for advanced glycation end products: a new biomarker in diagnosis and prognosis of chronic inflammatory diseases. Rheumatology (Oxford). 2009:48:1190-6.

43. Däbritz J, Friedrichs F, Weinhage T, Hampe J, Kucharzik T, Lügering A, et al. The functional -374 T/A polymorphism of the receptor for advanced glycation end products may modulate Crohn's disease. Am J Gastroent Liver Physiol. 2011;300:G823-32.

44. Yan SS, Wu ZY, Zhang HP, Furtado G, Chen X, Yan SF, et al. Suppression of experimental autoimmune encephalomyelitis by selective blockade of encephalitogenic T-cell infiltration of the central nervous system. Nat Med. 2003;9:287-93.

45. Lee SW, Park KH, Park S, Kim JH, Hong SY, Lee SK, et al. Soluble receptor for advanced glycation end products alleviates nephritis in (NZB/NZW) F1 mice. Arthritis Rheum. 2013;65:1902-12 
46. Renard C, Chappey O, Wautier MP, Nagashima M, Morser J, Scherrmann JM, et al. Recombinant advanced glycation end product receptor pharmacokinetics in normal and diabetic rats. Mol Pharmacol. 1997:52:54-62.

47. Chavakis T, Bierhaus A, Al-Fakhri N, Schneider D, Witte S, Linn T, et al. The pattern recognition receptor (RAGE) is a counterreceptor for leukocyte integrins: a novel pathway for inflammatory cell recruitment. J Exp Med. 2003;198:1507-15.

48. Roy CN, Semba RD, Sun K, Bandinelli S, Varadhan R, Patel KV, et al. Circulating selenium and carboxylmethyl-lysine, an advanced glycation end products, are independent predictors of anemia in older community-dwelling adults. Nutrition. 2012;28:762-6.

\section{Submit your next manuscript to BioMed Central} and take full advantage of:

- Convenient online submission

- Thorough peer review

- No space constraints or color figure charges

- Immediate publication on acceptance

- Inclusion in PubMed, CAS, Scopus and Google Scholar

- Research which is freely available for redistribution 